\title{
A GRÜSS' TYPE INTEGRAL INEQUALITY FOR MAPPINGS OF $r$-HÖLDER'S TYPE AND APPLICATIONS FOR TRAPEZOID FORMULA
}

\author{
SEVER SILVESTRU DRAGOMIR
}

\begin{abstract}
A new integral inequality of Grüss' type for mappings of $r$-Hölder's type and applications for trapezoid formula in Numerical Integration are given.
\end{abstract}

\section{Introduction}

In 1935, G. Grüss proved the following integral inequality:

$$
\left|\frac{1}{b-a} \int_{a}^{b} f(x) g(x) d x-\frac{1}{b-a} \int_{a}^{b} f(x) d x \cdot \frac{1}{b-a} \int_{a}^{b} g(x) d x\right| \leq \frac{1}{4}(\Phi-\phi)(\Gamma-\gamma)
$$

provided that $f$ and $g$ are two integrable functions on $[a, b]$ and satisfying the condition

$$
\phi \leq f(x) \leq \Phi \text { and } \gamma \leq g(x) \leq \Gamma \text { for all } x \in[a, b] .
$$

The constant $\frac{1}{4}$ is the best possible one and is achieved for $f(x)=g(x)=\operatorname{sgn}\left(x-\frac{a+b}{2}\right)$.

For other similar results, generalizations for positive linear functionals, discrete versions, determinantal versions etc. see the Chapter $X$ of the book [1] due to Mitrinović, Pečarić and Fink where further references are given.

In this paper we shall point out a new Grüss' type integral inequality for mappings of $r$-Hölder's type and apply it in connection with the trapezoid rule from Numerical Integration.

\section{The Results}

In this section we point out a Grüss' type inequality for mappings satisfying the condition of Hölder as follows

Theorem 2.1. Suppose that $f$ is of $r-H_{1}$-Hölder type and $g$ is of $s-H_{2}$-Hölder type, i.e.,

$$
|f(x)-f(y)| \leq H_{1}|x-y|^{r} \text { and }|g(x)-g(y)| \leq H_{2}|x-y|^{s}
$$

Received February 4, 1999.

1991 Mathematics Subject Classification. 26D15, 41A55.

Key words and phrases. Grüss' inequality, Numerical Integration. 
for all $x, y \in[a, b]$, where $H_{1}, H_{2}>0$ and $r, s \in(0,1]$ are fixed. Then we have the inequality:

$$
\begin{aligned}
& \left|\frac{1}{b-a} \int_{a}^{b} f(x) g(x) d x-\frac{1}{b-a} \int_{a}^{b} f(x) d x \cdot \frac{1}{b-a} \int_{a}^{b} g(x) d x\right| \\
\leq & \frac{H_{1} H_{2}(b-a)^{r+s}}{(r+s+1)(r+s+2)} .
\end{aligned}
$$

Proof. By the assumption (2.1) we have

$$
|(f(x)-f(y))(g(x)-g(y))| \leq H_{1} H_{2}|x-y|^{r+s}
$$

for all $x, y \in[a, b]$. Integrating on $[a, b]^{2}$ we get

$$
\begin{aligned}
\left|\int_{a}^{b} \int_{a}^{b}(f(x)-f(y))(g(x)-g(y)) d x d y\right| & \leq \int_{a}^{b} \int_{a}^{b}|(f(x)-f(y))(g(x)-g(y))| d x d y \\
& \leq H_{1} H_{2} \int_{a}^{b}|x-y|^{r+s} d x d y .
\end{aligned}
$$

Now, we observe that:

$$
\begin{aligned}
\int_{a}^{b} \int_{a}^{b}|x-y|^{r+s} d x d y & =\int_{a}^{b}\left(\int_{a}^{b}|y-x|^{r+s} d y\right) d x \\
& =\int_{a}^{b}\left(\int_{a}^{x}(x-y)^{r+s} d y+\int_{x}^{b}(y-x)^{r+s} d y\right) d x \\
& =\int_{a}^{b}\left[\frac{(x-a)^{r+s+1}+(b-x)^{r+s+1}}{r+s+1}\right] d x=\frac{2(b-a)^{r+s+2}}{(r+s+1)(r+s+2)}
\end{aligned}
$$

and as

$\frac{1}{2} \int_{a}^{b} \int_{a}^{b}(f(x)-f(y))(g(x)-g(y)) d x d y=(b-a) \int_{a}^{b} f(x) g(x) d x-\int_{a}^{b} f(x) d x \int_{a}^{b} g(x) d x$ we get the desired inequality $(2.2)$.

Remark 2.2. If $s=r=1$, i.e., in the case of Lipschitzian mappings, we have the following inequality [1]

$$
\left|\frac{1}{b-a} \int_{a}^{b} f(x) g(x) d x-\frac{1}{b-a} \int_{a}^{b} f(x) d x \cdot \frac{1}{b-a} \int_{a}^{b} g(x) d x\right| \leq \frac{L_{1} L_{2}}{12}(b-a)^{2},
$$

where $L_{1}$ and $L_{2}$ are the corresponding Lipschitz constants. 


\section{Applications for Trapezoid Formula}

In this section we point out some applications of the above results for the trapezoid rule as follows

Theorem 3.1. Let $f:[a, b] \rightarrow R$ be a differentiable mapping and assume that its derivative $f^{\prime}:(a, b) \rightarrow R$ is of $r$-Hölder's type on $(a, b)$, i.e.,

$$
\left|f^{\prime}(x)-f^{\prime}(y)\right| \leq H|x-y|^{r} \text { for all } x, y \in(a, b)
$$

where $r$ is fixed in $(0,1]$. Then we have the inequality

$$
\left|\frac{f(a)+f(b)}{2}-\frac{1}{b-a} \int_{a}^{b} f(x) d x\right| \leq \frac{H(b-a)^{r+1}}{(r+2)(r+3)} .
$$

Proof. Integrating by parts, we have

$$
\begin{aligned}
\int_{a}^{b}\left(x-\frac{a+b}{2}\right) f^{\prime}(x) d x & =\left.\left(x-\frac{a+b}{2}\right) f(x)\right|_{a} ^{b}-\int_{a}^{b} f(x) d x \\
& =(b-a) \frac{f(a)+f(b)}{2}-\int_{a}^{b} f(x) d x
\end{aligned}
$$

i.e., we have the identity

$$
\frac{f(a)+f(b)}{2}-\frac{1}{b-a} \int_{a}^{b} f(x) d x=\frac{1}{b-a} \int_{a}^{b}\left(x-\frac{a+b}{2}\right) f^{\prime}(x) d x .
$$

Define $f_{1}:[a, b] \rightarrow R, f_{1}(x)=x-\frac{a+b}{2}$ and $g_{1}:[a, b] \rightarrow R, g_{1}(x)=f^{\prime}(x)$. Then $f_{1}$ is of $s$ - $H_{1}$-Hölder's type with $s=1, H_{1}=1$. Applying Theorem 2.1 for $f_{1}$ and $g_{1}$ we get

$$
\begin{aligned}
& \left|\frac{1}{b-a} \int_{a}^{b}\left(x-\frac{a+b}{2}\right) f^{\prime}(x) d x-\frac{1}{b-a} \int_{a}^{b}\left(x-\frac{a+b}{2}\right) d x \cdot \frac{1}{b-a} \int_{a}^{b} f^{\prime}(x) d x\right| \\
\leq & \frac{H(b-a)^{r+1}}{(r+2)(r+3)} .
\end{aligned}
$$

Now, as

$$
\int_{a}^{b}\left(x-\frac{a+b}{2}\right) d x=0
$$

then the inequality (3.4) becomes, via the identity (3.3) the desired inequality (3.2).

The following approximation of the integral $\int_{a}^{b} f(x) d x$ holds.

Corollary 3.2. Suppose that $f$ is as above. If $I_{h}: a=x_{0}<x_{1}<\cdots<x_{n_{1}}<x_{n}=b$ is a partitioning of $[a, b]$ and $h_{i}:=x_{i+1}-x_{i}(i=0, \ldots, n-1)$ then we have

$$
\int_{a}^{b} f(t) d t=A_{T, I_{h}(f)}+R_{T, I_{h}}(f)
$$


where

$$
A_{T, I_{h}}(f)=\sum_{i=0}^{n-1} \frac{f\left(x_{i}\right)+f\left(x_{i+1}\right)}{2} h_{i},
$$

is the classical trapzoid rule and the remainder $R_{T, I_{h}}(f)$ is satisfying the estimation

$$
\left|R_{T, I_{h}}(f)\right| \leq \frac{H}{(r+2)(r+3)} \sum_{i=0}^{n-1} h_{i}^{r+2} .
$$

Proof. Applying Theorem 3.1 on the interval $\left[x_{i}, x_{i+1}\right](i=0, \ldots, n-1)$ we get

$$
\left|\left(x_{i+1}-x_{i}\right) \frac{f\left(x_{i}\right)+f\left(x_{i+1}\right)}{2}-\int_{x_{i}}^{x_{i+1}} f(t) d t\right| \leq \frac{H}{(r+2)(r+3)} h_{i}^{r+2}
$$

for all $i=0, \ldots, n-1$.

Summing the above inequalities and using the generalized triangle inequality we get the approximation formula (3.5) and the remainder is satisfying the estimation (3.7).

The following theorem concerning a perturbed trapezoid formula also holds.

Theorem 3.3. Let $f:[a, b] \rightarrow R$ be a twice differentiable mapping and assume that its second derivative $f^{\prime \prime}:(a, b) \rightarrow R$ is of $r$-H-Hölder's type on $(a, b)$ i.e.,

$$
\left|f^{\prime \prime}(x)-f^{\prime \prime}(y)\right| \leq H|x-y|^{r} \text { for all } x, y \in(a, b)
$$

where ris fixed in $(0,1]$. Then we have the inequality

$$
\left|\frac{1}{b-a} \int_{a}^{b} f(x) d x-\frac{f(a)+f(b)}{2}+\frac{(b-a)}{12}\left(f^{\prime}(b)-f^{\prime}(a)\right)\right| \leq \frac{H(b-a)^{r+2}}{2(r+2)(r+3)} .
$$

Proof. Integrating by parts, we can state that

$$
\begin{aligned}
\int_{a}^{b}(x-a)(b-x) f^{\prime \prime}(x) d x & =\left[(x-a)(b-x) f^{\prime}(x)\right]_{a}^{b}-\int_{a}^{b}[(a+b)-2 x] f^{\prime}(x) d x \\
& =\int_{a}^{b}[2 x-(a+b)] f^{\prime}(x) d x=\left.f(x)[2 x-(a+b)]\right|_{a} ^{b}-2 \int_{a}^{b} f(x) d x,
\end{aligned}
$$

from where we get the equality

$$
\int_{a}^{b}(x) d x=\frac{f(a)+f(b)}{2}(b-a)-\frac{1}{2} \int_{a}^{b}(x-a)(b-x) f^{\prime \prime}(x) d x .
$$

Consider the mapping $f_{1}:[a, b] \rightarrow R, f_{1}(x)=(x-a)(b-x)$. Then $f_{1}^{\prime}(x)=(a+b)-2 x$ and then $\left|f_{1}^{\prime}(x)\right| \leq b-a$ for all $x \in[a, b]$, and then $f_{1}$ is of $s$ - $H_{1}$-Hölder's type with $s=1$, $H_{1}=b-a$. Consider also $g_{1}:(a, b) \rightarrow R, g_{1}(x)=f^{\prime \prime}(x)$ which is of $r$-H-Hölder's type. 
Applying Theorem 2.1 for $f_{1}$ and $g_{1}$ we get:

$$
\begin{aligned}
& \left|\frac{1}{b-a} \int_{a}^{b}(x-a)(b-x) f^{\prime \prime}(x) d x-\frac{1}{b-a} \int_{a}^{b}(x-a)(b-x) d x \cdot \frac{1}{b-a} \int_{a}^{b} f^{\prime \prime}(x) d x\right| \\
\leq & \frac{H(b-a)^{r+2}}{(r+2)(r+3)}
\end{aligned}
$$

Now, as

$$
\int_{a}^{b}(x-a)(b-x) d x=\frac{(b-a)^{3}}{6} \text { and } \int_{a}^{b} f^{\prime \prime}(x) d x=f^{\prime}(b)-f^{\prime}(a)
$$

we get from (3.11) that

$$
\left|\frac{1}{b-a} \int_{a}^{b}(x-a)(b-x) f^{\prime \prime}(x) d x-\frac{b-a}{6}\left(f^{\prime}(b)-f^{\prime}(a)\right)\right| \leq \frac{H(b-a)^{r+2}}{(r+2)(r+3)} .
$$

Using the identity (3.10), the above inequality becomes the desired result (3.9).

The following quasi-trapezoid composite formula holds.

Corollary 3.4. Let $f$ be as in the above theorem. If $I_{h}$ is a partitioning of $[a, b]$, then we have

$$
\int_{a}^{b} f(t) d t=A_{Q T, I_{h}}(f)+R_{Q T, I_{h}}(f)
$$

where

$$
A_{Q T, I_{h}}(f)=\sum_{i=0}^{n-1} \frac{f\left(x_{i}\right)+f\left(x_{i+1}\right)}{2} h_{i}-\frac{1}{12} \sum_{i=0}^{n-1}\left(f^{\prime}\left(x_{i+1}-f^{\prime}\left(x_{i}\right)\right) h_{i}^{2}\right.
$$

is a quasi-trpezoid rule and the remainder $R_{Q T, I_{h}}(f)$ is satisfying the estimation

$$
\left|R_{Q T, I_{h}}(f)\right| \leq \frac{H}{2(r+2)(r+3)} \sum_{i=0}^{n-1} h_{i}^{r+3} .
$$

The proof follows by the above theorem and we shall omit the details.

\section{References}

[1] D. S. Mitrinović, J. E. Pečarić and A. M. Fink, Classical and New Inequalities in Analysis, Kluwer Academic Publishers, Dordrecht, 1993.

Department of Mathematics, Victoria University of Technology, PO BOX 14428, Melbourne City, MC 8001 Australia.

E-mail: sever@matilda.vu.edu.au 